EPJ Web of Conferences 49, 11003 (2013)

DOI: $10.1051 /$ epjconf/20134911003

(C) Owned by the authors, published by EDP Sciences, 2013

\title{
Prospects for physics at high luminosity with CMS
}

\author{
João Varela ${ }^{1}$ \\ Laboratório de Instrumentação e Partículas \\ Av. Elias Garcia, 1000-149 Lisbon, Portugal \\ Deputy Spokesperson, CMS Experiment \\ E-mail: joao.varela@cern.ch
}

\begin{abstract}
The precision measurements of the properties of the recently discovered Higgs-like boson will be central to the future LHC physics program. In parallel the search for New Physics beyond the SM will continue. Higher luminosity will extend the mass reach and allow sensitive searches for possible subtle signatures for new physics. In this paper we review the potential sensitivity of CMS to a selection of relevant future physics scenarios accessible with the LHC upgrades and a correspondingly upgraded CMS detector.
\end{abstract}

\footnotetext{
${ }^{1}$ On behalf of the CMS Collaboration
}

\section{Introduction}

With the first $10 \mathrm{fb}^{-1}$ of data at 7 and $8 \mathrm{TeV}$ ATLAS and CMS have dramatically changed the picture of the search for a standard model (SM) Higgs boson, excluding all masses below $600 \mathrm{GeV}$ except for a narrow mass interval around $125 \mathrm{GeV}$. Here both ATLAS and CMS observe a new boson with a significance of about five sigma [1,2]. Direct searches for physics beyond the SM have not been successful yet. Gluinos and squarks with masses below $1 \mathrm{TeV}$ are excluded in simple Supersymmetry models while scenarios with $3^{\text {rd }}$ generation squarks below $1 \mathrm{TeV}$ are still compatible with the data. New gauge bosons with SM couplings are excluded below $2.5 \mathrm{TeV}$.

Following the discovery of a Higgs-like boson, the precision measurements of the properties of this new particle will be central to the LHC physics program. The search for New Physics beyond the SM will continue. Higher luminosity and higher energies will both extend the mass reach and allow sensitive searches for possible subtle signatures for new physics.

In this paper we review the potential sensitivity of CMS to a selection of relevant future physics scenarios accessible with the LHC upgrades and a correspondingly upgraded CMS detector [3]. A more complete account of the CMS expected performance can be found in [4].

\section{CMS upgrade program}

Starting with the first long shutdown, and leading up to the HL-LHC, a number of incremental CMS detector upgrades are underway. We designate by Phase- 1 the upgrades to be implemented before or during the second long shutdown. There are three major projects planned in
Phase-1: the replacement of the pixel detector with a four-layer high-data-rate design, a new L1-Trigger system with higher granularity and additional processing capabilities, and an upgrade to the photo-detectors and electronics of the hadron calorimeters (HCAL) to reduce background signals and improve measurement of jets and missing-energy at high pile-up.

All components of the L1-Trigger system will be upgraded, namely the calorimeter, muon and global trigger systems. The new system uses the full granularity of the calorimeter trigger tower information. New algorithms for electron/photon, jet, tau and missing $E_{T}$ will be implemented in powerful FPGAs of the latest generation. Event-by-event pile-up estimation and subtraction will be implemented, considerably improving the performance of the calorimeter trigger algorithms. In addition, the upgraded system will permit the combination of calorimeter cluster information with L1 muon tracks allowing requirements on muon isolation to be implemented at L1-trigger level. New muon track finders will increase the efficiency and purity of the L1 muon candidates. Sophisticated kinematical correlations between trigger objects will be available in the new global trigger system, including the computation of invariant mass. The improved efficiency and discrimination of the upgraded system will enable CMS to maintain the present trigger acceptance compatible with a L1-trigger rate of $100 \mathrm{kHz}$, for luminosities up to $2 \times 10^{34} \mathrm{~cm}^{-2} \mathrm{~s}^{-1}$.

The innermost tracking detector, the pixel detector, will be replaced with an improved device that adds a fourth layer to its design [5]. In addition to improving the 
overall quality and robustness of track reconstruction, the upgraded pixel system will provide substantially improved b-tagging capability.

The overall configuration of the new detector is shown in figure 1 . The first barrel layer is moved closer to the interaction point, by $14 \mathrm{~mm}$, at a radius of $30 \mathrm{~mm}$; this will improve the track Impact Parameter (IP) resolution. The radius of the outermost layer, now the fourth layer, increases to $160 \mathrm{~mm}$, closer to the Tracker Inner Barrel (TIB) layers; this will reduce the rate of fake tracks and mitigate inefficiencies in the TIB. The new detector will have 123 million pixels, almost twice the present system. The smaller inner radius of the first layer requires the installation of a new beam pipe.

In the new pixel detector the barrel connectors and optical links will be positioned at the end of the service supply tubes. Along with the use of a $\mathrm{CO}_{2}$ cooling system and lightweight support structures, this change will allow the new detector to have less material in the tracking acceptance than the current one, even with one additional layer and disk.

The performance of the pixel detector is characterized by the efficiency to reconstruct tracks, the rate of fakes and the precision of the track IP measurement. The detailed simulations performed for different rate and pileup conditions demonstrate that the new device will have better performance at pile-up of 50 collisions than the current detector at pile-up of 25 collisions.

The upgrades of the hadron calorimeter systems are based on the replacement of the photomultipliers currently used in these calorimeters [6]. For the barrel and endcap systems, the newly-proven technology of Silicon Photomultipler (SiPM) will be used. The new photo-detectors with newly designed readout chips provide an order of magnitude higher signal-to-noise ratio in the barrel and endcap calorimeters, allowing longitudinal segmentation of the calorimeter without introducing excessive noise. In the forward calorimeter new multi-anode PMTs provide redundant sampling of the light from each calorimeter cell.

The depth segmentation of the hadronic calorimeter depicted in figure 2 provides better tracking of hadronic shower development. This allows to significantly reduce the effects of pile-up in the measurement of jets and missing $\mathrm{E}_{\mathrm{T}}$, particularly for particle flow algorithms in high occupancy events. Simulation studies of particleflow behavior at high pile up show large numbers of anomalous hits and large clusters of energy as the particle flow algorithms can no longer distinguish individual high-energy particle showers. The addition of depth segmentation eliminates the anomalous hits and improves the association of clusters and tracks which is crucial for particle-flow techniques.

For the HL-LHC, a number of additional, major, upgrades are foreseen. These are called Phase-2 upgrades. The tracking system will have to be completely replaced since the current one will reach the limit of the expected lifetime under radiation. We envisage that the upgraded tracker, in addition to providing improved tracking capability in a very high density environment and presenting substantially lower material, will also provide a L1 Tracking Trigger, reconstructing charged particles with $\mathrm{p}_{\mathrm{T}}$ greater than $2.5 \mathrm{GeV}$ and thereby substantially improving the overall L1-trigger functionality. In addition, CMS is examining a possible upgrade to the data acquisition and high level trigger systems, which would allow up to $1 \mathrm{MHz} \mathrm{L} 1$ read-out rate and up to $10 \mathrm{kHz}$ event storage rate.

Studies are underway to ensure that precision electromagnetic calorimetry and robust jet and missing transverse energy reconstruction capability are maintained at the HL-LHC. In this context, the replacement of the end-cap and forward calorimeters is foreseen, as well as increased tracking coverage to extend particle flow reconstruction up to a pseudorapidity of four. In addition, studies are considering a possible electromagnetic pre-shower system, capable both of providing pointing information for the unambiguous reconstruction of Higgs to two-photon events, as well as very high time-of-flight resolution for pile-up mitigation.

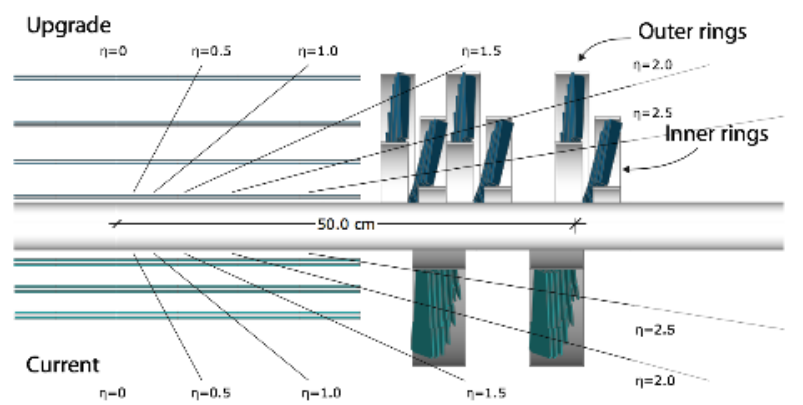

Fig. 1. Conceptual layout of pixel detector comparing the different layers and disks in the current and upgrade detectors.

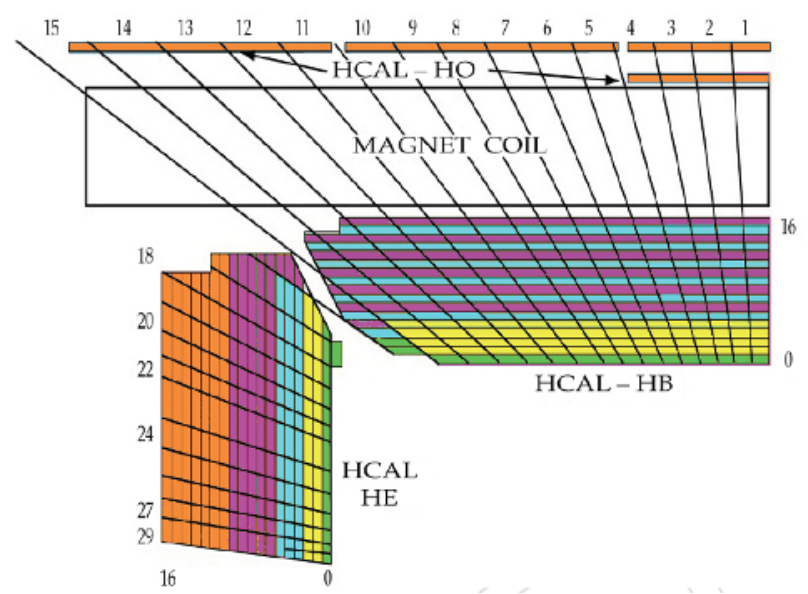

Fig. 2. Proposed depth segmentation structure for the HB and HE calorimeters, made possible by the use of SiPM photo detectors.

\subsection{Physics performance of Phase-1 Upgrade}

The improvements expected with the Phase-1 upgrade for representative physics signals have been evaluated at a luminosity of $2 \times 10^{34} \mathrm{~cm}^{-2} \mathrm{~s}^{-1}$ with $25 \mathrm{~ns}$ bunch spacing. Fully simulated Monte Carlo events of Higgs decay channels have been produced and analyzed with the same analysis code that is used for the 2012 data, with very limited modifications to adapt to the different beam conditions. The MC samples have been simulated with center-of-mass energy of $14 \mathrm{TeV}$ and 50 overlapping 
events per bunch-crossing. This last value is twice as large as the typical pile-up at the beginning of a fill in the 2012 data taking.

The studies included the associated production $\mathrm{ZH}$ with the $\mathrm{Z}$ decaying to muons and the Higgs boson decaying to b-quark pair, which will benefit significantly from the increased muon tracking efficiency and btagging efficiency provided by the upgraded pixel detector. A similar analysis with the $\mathrm{Z}$ decaying to electrons benefits in addition from the decreased material in the tracking volume. Both lepton channels $(\mu \mu$, ee) show a gain of $65 \%$ in signal efficiency with the upgraded system.

A second study evaluates the improvement of the Higgs boson search in the $\mathrm{H} \rightarrow \mathrm{ZZ} \rightarrow 41 \quad(1=\mathrm{e}, \mu)$ channel. The analysis is optimized for a Higgs boson in the mass range $110 \mathrm{GeV}<\mathrm{m}_{\mathrm{H}}<160 \mathrm{GeV}$ for center-of-mass energies of 7-8 TeV. The analysis performance is quite sensitive to the lepton tracking and isolation efficiency. The Monte-Carlo results show an improvement in signal efficiency of $41 \%$ in the four-muon channel and of $51 \%$ in the four-electron channel.

The physics improvements due to the HCAL upgrade are illustrated by an analysis optimized for SM Higgs boson production through vector boson fusion (VBF) and Higgs final state with tau pairs. A comparative study of the performance of VBF-tagging associated to the e $\mu$ taupair final state, was made with the upgrade detector (combined HCAL and pixel upgrades) and the present detector. The improvements in jet reconstruction and identification in the upgrade scenario, translate in significantly lower rates for selecting jets from pile-up, in an improvement in $\mathrm{m}_{\tau \tau}$ resolution of $25 \%$ and in a substantial increase of the signal efficiency in this channel. The selection efficiency for events passing the VBF Higgs selection normalized to the inclusive selection efficiency is found to be $11 \%$ in the upgrade scenario and $4.6 \%$ in the current detector. The Phase- 1 upgrade thus provides a potential improvement in the efficiency for a VBF signal by a factor of 2.4.

\section{Physics projections}

In this section we make the assumption that future CMS upgrades will provide at higher energy and luminosity the same level of detector and trigger performances we achieve with the current detector in the 2012 data taking period. This assumption is validated by the recent studies of the physics performance with the proposed upgrades of the pixel detector and of the hadron calorimeter, described in the previous section.

The results obtained show no degradation, and in several occasions a significant improvement, of the physics performances of the detector at instantaneous luminosity of $2 \times 10^{34} \mathrm{~cm}^{-2} \mathrm{~s}^{-1}$ with 50 pile-up events. This gives us confidence that the projections presented here for $300 \mathrm{fb}^{-1}$ are realistic and could possibly be improved by optimizing the analysis to the new beam conditions.

A full simulation of the Phase-2 CMS detector for HL-LHC is not performed at this time, and consequently the accuracy of further extrapolation to $3000 \mathrm{fb}^{-1}$ is more uncertain. Many of the measurements one would like to perform at HL-LHC are systematics limited and while statistical uncertainties can be reliably estimated, it is not possible to know exactly how systematic uncertainties will evolve. For the HL-LHC, a number of additional, major, upgrades are foreseen as described in the previous section. However, the future configuration of the CMS detector is not yet fully established.

\subsection{Higgs boson characterization}

The precise measurement of the properties of the new boson is of utmost importance. The key properties are the couplings to each fermion and boson, which are predicted by the SM. A variety of attempts exist to estimate the sensitivity of Higgs coupling measurements at the LHC and future electron-positron collider [7-10]

The mass and the spin-state of the new particle can be accurately determined using the dataset recorded in 2012. To further characterize the properties of the new particle we focus on the measurements of its couplings. In order to estimate the precision of future Higgs coupling measurements we scale the signal and background cross sections considered in the searches discussed in [1] to the LHC scenarios of $300 \mathrm{fb}^{-1}$ and $3000 \mathrm{fb}^{-1}$ at $14 \mathrm{TeV}$.

We present extrapolations under three uncertainty scenarios. In the first one all systematic uncertainties are kept unchanged (scenario-1). In the second one the theoretical uncertainties are scaled by a factor of $1 / 2$, while other systematical uncertainties are scaled by the square root of the integrated luminosity (scenario-2). The comparison of the two uncertainty scenarios indicates a range of possible future measurements. The uncertainties on the expected signal strength and the Higgs couplings is also shown without theoretical uncertainties to illustrate the effect of those uncertainties (scenario-3).

\subsubsection{Expectations with luminosity $300 \mathrm{fb}^{-1}$}

When testing the compatibility with the SM we adopt an effective theory approach. We hereby add modifiers to an effective Lagrangian which can be matched at leading order to the SM Higgs boson couplings. The fit estimates the expected uncertainty on the overall signal strength, $\mu=\sigma / \sigma_{\mathrm{SM}}$, e.g. the precision of Higgs production cross sections times branching fraction, and is summarized in figure 2. The estimated precision is of the order of 10$15 \%$.

Assuming that all boson and all fermion couplings are modified in the same way, we can perform a fit for $\mathrm{C}_{\mathrm{V}}$ and $\mathrm{C}_{\mathrm{F}}$, the couplings respectively to bosons and to fermions. This benchmark uses the explicit assumption that no new physics is present and there are no additional loops in the production or decay of the Higgs boson. Figure 3 shows the one and two sigma contours for the measurement of $\mathrm{C}_{\mathrm{V}}$ and $\mathrm{C}_{\mathrm{F}}$. A precision of 10 percent is expected at $\sqrt{\mathrm{s}}=14 \mathrm{TeV}$ and for an integrated luminosity of $300 \mathrm{fb}^{-1}$.

To test for more subtle deviations from the SM, we increase the number of degrees of freedom in the couplings fit to six and fit for $\kappa \gamma, \kappa_{\mathrm{V}}, \kappa_{\mathrm{g}}, \kappa_{\mathrm{b}}, \kappa_{\mathrm{t}}$ and $\kappa \tau$, representing the couplings to photons, to the $\mathrm{W}$ and $\mathrm{Z}$ bosons, to gluons, to bottom and top quarks, and to the 
tau lepton. The total width of the Higgs boson is constrained assuming no invisible Higgs decays. The uncertainties on the projections obtained with $300 \mathrm{fb}^{-1}$ are summarized in figure 4.

\section{CMS Projection}

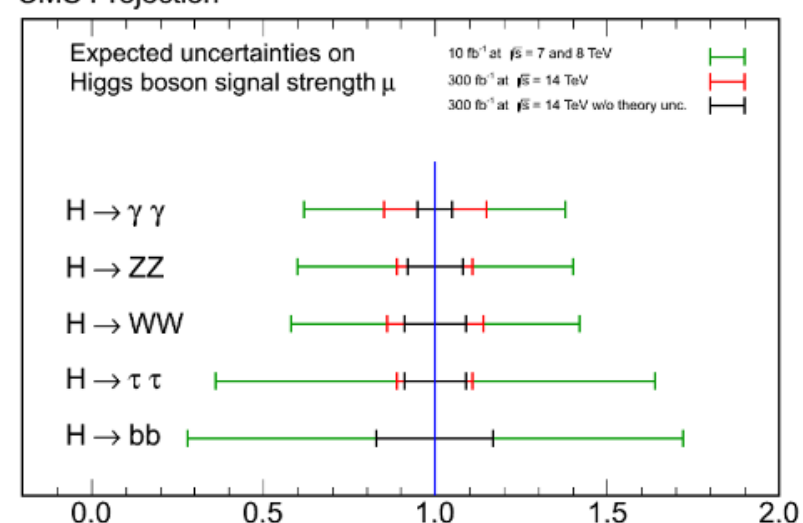

Fig. 2. Estimated precision of the signal strength determination for a SM Higgs boson. The projections assume $\sqrt{s}=14 \mathrm{TeV}$ and an integrated luminosity of $300 \mathrm{fb}^{-1}$. They are shown assuming the current systematics uncertainties (Scenario 1, red error bars) and neglecting the systematic uncertainties from theory (Scenario 3, black error bars) and are compared to the expected uncertainties of the measurement with $10 \mathrm{fb}^{-1}$ at $\sqrt{\mathrm{s}}=$ 7 and $8 \mathrm{TeV}$ (green error bars).

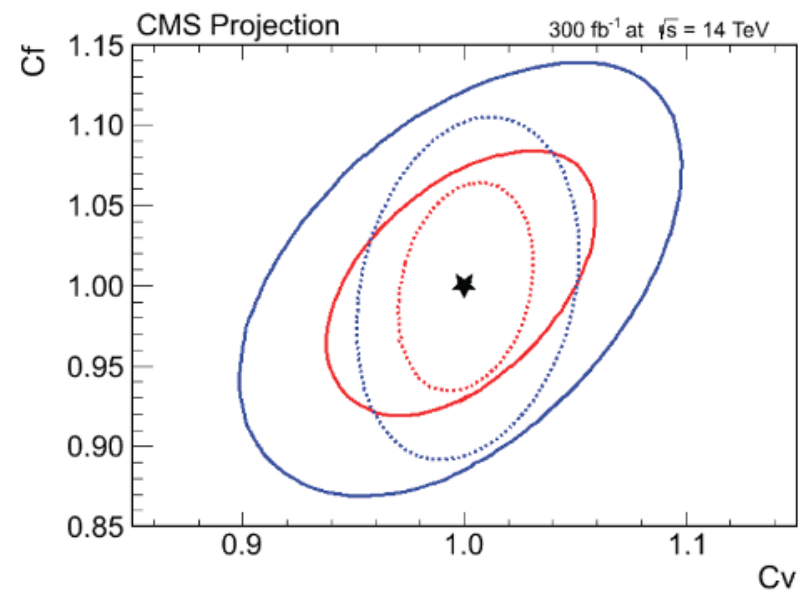

Fig. 3. Estimated precision on the measurement of $\mathrm{C}_{V}$ and $\mathrm{C}_{\mathrm{F}}$ as one (red) and two (blue) standard deviation (i.e., $68 \%$ and $95 \%$ ) confidence level contours. The projections (solid contours) assume $\sqrt{\mathrm{s}}=14 \mathrm{TeV}$ and an integrated luminosity of $300 \mathrm{fb}^{-1}$ under Scenario 1, while the dotted lines show the projections neglecting the systematic uncertainties from theory (Scenario 3).

\subsubsection{Expectations with luminosity $3000 \mathrm{fb}^{-1}$}

In order to obtain the asymptotic precision with which the Higgs couplings can be measured at the LHC, we also calculated the expected uncertainties on the couplings with a data sample of $3000 \mathrm{fb}^{-1}$. In these calculations we compare scenario-1 (unchanged systematic uncertainties) and scenario-2 (experimental systematical uncertainties scaled by the square root of the integrated luminosity and theoretical systematics scaled by a factor 2). The latter can be justified by noting that the experimental uncertainties are ultimately data driven. On the other hand, there are numerous examples from LEP and the Tevatron where optimistic projections were realized and even better performances were achieved.

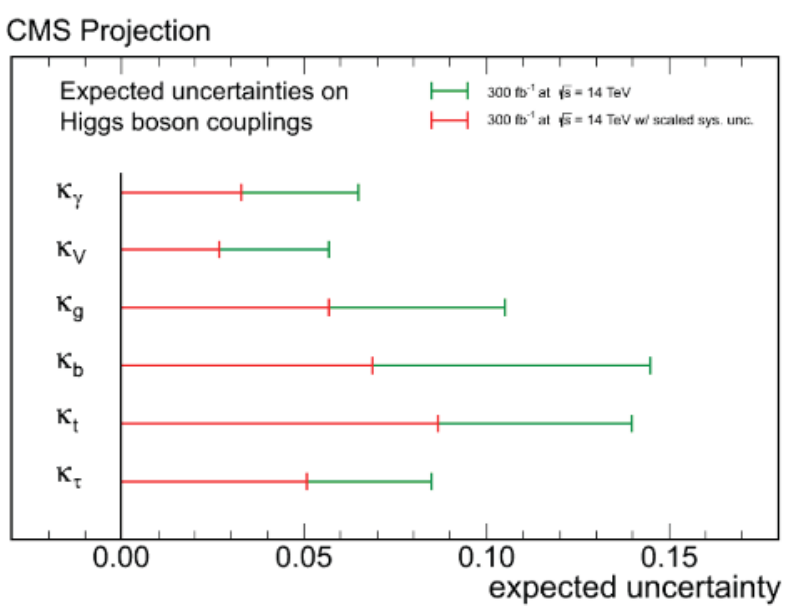

Fig. 4. Estimated precision on the measurements of $\kappa \gamma, \kappa_{V}, \kappa_{g}$, $\kappa_{\mathrm{b}}, \kappa_{\mathrm{t}}$ and $\kappa \tau$. The projections assume $\sqrt{\mathrm{s}}=14 \mathrm{TeV}$ and an integrated dataset of $300 \mathrm{fb}^{-1}$. The green error bars correspond to Scenario1, while the red error bars correspond to Scenario 2 described in the text.

Table 1. Achievable precision on the measurements of $\kappa \gamma, \kappa_{V}$, $\kappa_{\mathrm{g}}, \kappa_{\mathrm{b}}, \kappa_{\mathrm{t}}$ and $\kappa \tau$. These values are obtained at $\sqrt{\mathrm{s}}_{\mathrm{s}}=14 \mathrm{TeV}$ using an integrated dataset of 300 and $3000 \mathrm{fb}^{-1}$. The results are shown for two uncertainty scenarios. In the first one (Scenario 1) all systematic uncertainties are kept the same as in the $8 \mathrm{TeV}$ analysis. In the second one (Scenario 2) the theoretical uncertainties are scaled by a factor of $1 / 2$, while other systematical uncertainties are scaled by the square root of the integrated luminosity.

\begin{tabular}{|c|c|c|c|c|}
\hline \multirow{2}{*}{ Coupling } & \multicolumn{4}{|c|}{ Uncertainty (\%) } \\
\cline { 2 - 5 } & \multicolumn{2}{|c|}{$300 \mathrm{fb}^{-1}$} & \multicolumn{2}{c|}{$3000 \mathrm{fb}^{-1}$} \\
\cline { 2 - 5 } & Scenario 1 & Scenario 2 & Scenario 1 & Scenario 2 \\
\hline \hline$\kappa_{\gamma}$ & 6.5 & 5.1 & 5.4 & 1.5 \\
$\kappa_{V}$ & 5.7 & 2.7 & 4.5 & 1.0 \\
$\kappa_{g}$ & 11 & 5.7 & 7.5 & 2.7 \\
$\kappa_{b}$ & 15 & 6.9 & 11 & 2.7 \\
$\kappa_{t}$ & 14 & 8.7 & 8.0 & 3.9 \\
$\kappa_{\tau}$ & 8.5 & 5.1 & 5.4 & 2.0 \\
\hline
\end{tabular}

The results obtained are shown in Table1. These numbers should not be interpreted as precise projections, but rather as the lower and upper bounds on the uncertainty that could be achieved with HL-LHC.

Ideally, the measurement of couplings should be extended to first and second generation fermions. This is a particularly difficult task at the LHC. Previous studies have shown that the Higgs decay to a pair of muons can be observed in gluon-gluon fusion and via vector boson fusion production [11-13]. Assuming the current performance of the detector, CMS confirms these studies and estimates that in both production modes at HL-LHC, an excess with a significance of approximately $5 \sigma$ can be observed, allowing a measurement of the H $\mu \mu$ coupling with a precision of better than $10 \%$.

Perhaps the most important measurement after the discovery of the Higgs boson is the measurement of the Higgs potential itself. This can be probed with the study 
of multiple Higgs boson production. Previous studies [1419] have shown that a measurement of multiple Higgs boson production is possible with a dataset of $3000 \mathrm{fb}^{-1}$ at the LHC. The SM cross section for di-Higgs production is 33 (206) fb at 14 (33) TeV. Modifying the Higgs selfcoupling to zero or to twice the SM value increases or reduces the cross section by a factor of two. The theoretical uncertainty is dominated by the scale uncertainties and is of the order of $15-20 \%$. Promising final states are those that allow a precision measurement of the mass of one of the two Higgs bosons, e.g. the bb $\gamma \gamma$ or bbu final states, which allow to reduce the experimental backgrounds.

\subsection{Searches for new physics}

\subsubsection{Searches with missing energy}

In case the new particle with a mass of $125 \mathrm{GeV}$ is confirmed as the standard model Higgs boson, finding a solution to the stability of the Higgs mass to large quantum corrections becomes arguably the major issue in particle physics. Natural supersymmetry is one of the most effective and popular solutions to this problem, however no signal of supersymmetry has been seen yet at LHC. It is important to extend the mass reach and the scope of these searches and to find or exclude natural supersymmetry.

An Higgs boson mass of $125 \mathrm{GeV}$ may point to a rather heavy supersymmetry mass spectrum. Simple extrapolations of the present CMS searches for supersymmetry [20] are obtained scaling with luminosity and taking into account the change of cross sections with the collision energy. Cross sections have been computed with Pythia8 and the same signal efficiency as in the present searches is assumed. This is a reasonable hypothesis since the mass reach has a mild dependence on efficiency.

We studied three different scenarios: generic squark and gluinos search, a scenario where stops and sbottoms are the lightest squarks, and a search for SUSY partners of electroweak gauge bosons (EWKinos). Figure 5 shows the result of this study. The mass reach is consistent with the results presented in figure 13 of [11] within 100 $\mathrm{GeV}$.

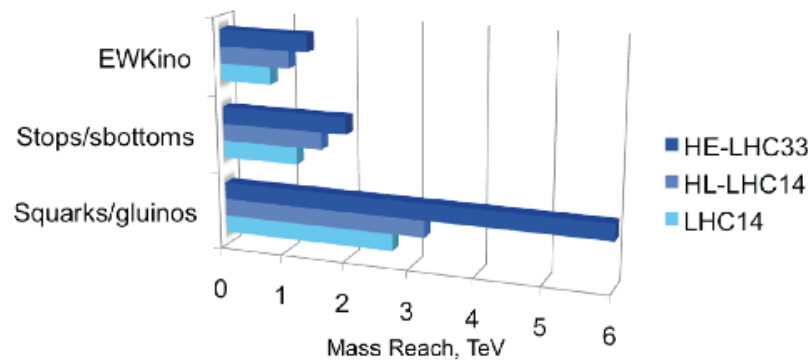

Fig. 5. Estimated reach of searches for supersymmetry.

LHC can find generic squarks and gluinos up to 2.7 $\mathrm{TeV}$, and HL-LHC increases the limit to $3.2 \mathrm{TeV}$. Going to higher energy is a major improvement and the sensitivity of HE-LHC reaches $6 \mathrm{TeV}$. The reach for stop and sbottom squarks assumes that only the lightest stop/sbottom is directly produced. The sensitivity to direct stop/sbottom production is up to $1.2 \mathrm{TeV}$ in $\mathrm{LHC}$, 1.6 TeV in HL-LHC and $2 \mathrm{TeV}$ in HE-LHC. EWKinos have lower cross sections. LHC can find charginos or neutralinos up to $0.8 \mathrm{TeV}$, HL-LHC extends this limit to $1.1 \mathrm{TeV}$ and HE-LHC reach is up to $1.4 \mathrm{TeV}$.

\subsubsection{Searches for new resonances}

Excluding natural supersymmetry at the LHC makes even more important to search for alternative solutions of the hierarchy problem. One possibility is the RS model of Extra Dimensions [21] that predicts also the existence of a new resonance, the Kaluza-Klein graviton.

The searches for new resonances will profit from the increase in the energy of the center of mass at HE-LHC. As representative example we studied the search for a generic resonance called $\mathrm{Z}^{\prime}$ decaying into an electron pair at HE-LHC, one of the possible decay modes of the Kaluza-Klein graviton. We have not considered the decay into a muon pair since muon identification becomes more difficult and the resolution on muon momentum degrades with energy. With electron energy resolution and electron identification similar to that which we have today, we obtain the curve shown in figure 6 where the expected limit on cross section times branching fraction is shown as function of the mass of the $Z^{\prime}$. These limits can be interpreted in the context of models predicting narrow resonances as $Z_{\text {SSM }}^{\prime}$ with standard model-like couplings or $Z^{\prime} \psi$ expected in grand unified theories giving lower mass boundaries around $10 \mathrm{TeV}$. The limit on the Kaluza-Klein graviton is $4.5(9.4) \mathrm{TeV}$ when the coupling parameter is $k / M_{P l}=0.01(0.1)$.

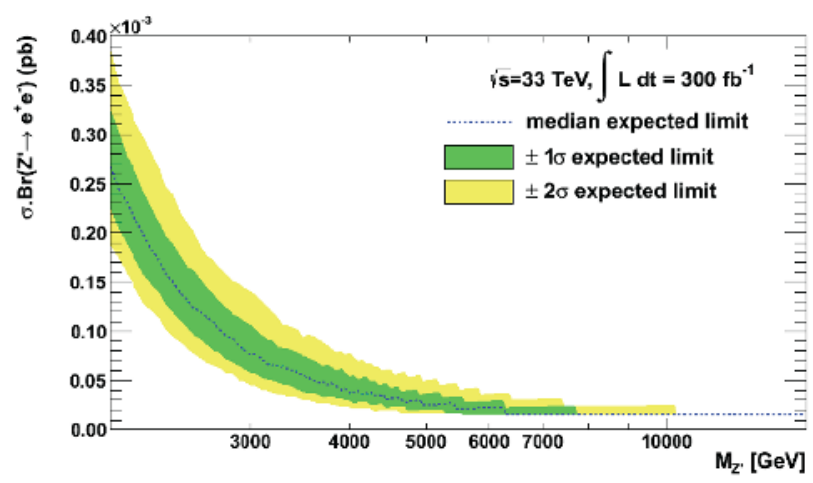

Fig. 6. Upper limits on the production cross section times branching fraction into electron pairs as a function of resonance mass $\mathrm{M}$.

Many models beyond the SM predict the existence of leptoquarks (LQ), hypothetical particles that carry both baryon number and lepton number and couple to both quarks and leptons. The mass reach of the searches for LQ will profit from the increase of the center of mass energy, but also from the large dataset collected at HLLHC. We have extrapolated the CMS search [22] in the eejj channel at $\sqrt{\mathrm{s}}=7 \mathrm{TeV}$, scaling the main backgrounds of the analysis with parton luminosity ratios and comparing it to the expected yield of scalar LQ (assuming 100\% branching fraction to ej and a selection efficiency of $50 \%$ as in the present analysis). The expected limit is computed for a working point scenario yielding $\mathrm{S} / \mathrm{B} \sim 1$. The results of this simple extrapolation 
give a mass reach of the leptoquark search in the eejj channel of 1.7, 2.3 and $3.5 \mathrm{TeV}$, respectively at LHC, HL-LHC and HE-LHC. These values can be compared to the present exclusion in this channel of $0.8 \mathrm{TeV}$.

\section{Conclusions}

The exploration of the full potential of the upgraded LHC is the highest priority for high-energy physics in the next two decades. Both the high-luminosity LHC upgrade and the potential high-energy upgrade at a later time would contribute greatly to our understanding of Nature.

The detailed characterization of the newly discovered $125 \mathrm{GeV}$ boson can be achieved by the CMS experiment through precision measurements of its mass and couplings to fermions and bosons, as well as the selfcoupling, with integrated luminosities of $300 \mathrm{fb}^{-1}$ at LHC and $3000 \mathrm{fb}^{-1}$ at HL-LHC. These measurements would allow to either prove that it is the SM Higgs boson or, if it is not, fully explore the electroweak symmetry breaking (EWSB) mechanism and uncover the true nature of the observed particle.

The mass reach of direct searches for new particles will be considerably extended at higher luminosities and center of mass energy, in particular the searches for SUSY squarks and gluinos, as well the search for new heavy resonances predicted in many extensions of the SM.

\section{References}

1. CMS Collaboration, Phys.Lett.B 716 (2012) 30-61

2. ATLAS Collaboration, Phys.Lett.B 716 (2012) 1-29

3. The CMS Collaboration et al 2008 JINST 3 S08004 doi:10.1088/1748-0221/3/08/S08004

4. CMS Collaboration, CMS-NOTE-2012-06

5. CMS Collaboration, CERN-LHCC-2012-015

6. CMS Collaboration, CERN-LHCC-2012-016

7. J.R.Espinosa, C.Grojean, M.Muhlleitner, M.Trott, JHEP 05 (2012) 097.

8. D.Carmi, A.Falkowski, E.Kuflik, T.Volansky, JHEP 07 (2012) 136.

9. A.Azatov, R.Contino, J.Galloway, JHEP 04 (2012) 127.

10. M.Klute, R.Lafaye, T.Plehn, M.Rauch, D.Zerwas, Phys. Rev. Lett. 109, (2012) 101801.

11. F. Gianotti, M.L. Mangano, T. Virdee et al, Eur.Phys.J. C39, 293 (2005).

12. T.Plehn, D.Rainwater, Phys. Lett. B 520, 108 (2001).

13. T.Han, B.McElrath, Phys. Lett. B 528, 81 (2002)

14. W.N.Glover, J.J.van der Bij, Phys. Lett. B 219, 488 (1989).

15. S.Dawson, S.Dittmaier, M.Spira, Phys. Rev. D 58, 115012 (1998).

16. A.Djouadi, W.Kilian, M.Muehlleitner, P.M.Zerwas, Eur. Phys. J. C 10, 45 (1999).

17. U.Baur, T.Plehn, D.Rainwater, Phys. Rev. Lett. 89, (2002) 151801; Phys. Rev. D 67, 033003(2003); Phys. Rev. D 68, 033001 (2003); Phys. Rev. D 69, 053004 (2004).

18. T.Binoth, S.Karg, N.Kauer, R.Rueckl, Phys. Rev. D 74, 113008 (2006).
19. M.J.Dolan, C.Englert, M.Spannowsky, arXiv: 1206. 5001.

20. CMS searches for supersymmetry can be found at https://twiki.cern.ch/twiki/bin/view/CMSPublic/Physi csResultsSUS.

21. L. Randall and R. Sundrum, Phys. Rev. Lett. 83, 3370 (1999).

22. CMS Collaboration, Phys. Rev. D 86 (2012) 052013. 